\title{
Sistema experto para la SGTI en la empresa Sion Global Solutions
}

\section{Expert system for the ITSM in the company Sion Global Solutions}

David Flores Zafra

(D) https://orcid.org/0000-0001-5846-325X

Universidad Norbert Wiener, Perú

Victoria Gardi Melgarejo

Universidad Norbert Wiener, Perú

Autor para correspondencia: david.flores@uwiener.edu.pe; victoria.gardi@uwiener.edu.pe Fecha de recepción: 23 de septiembre de 2020 - Fecha de aceptación: 11 de diciembre de 2020

\section{Resumen}

En la empresa, se determinó que el nivel de madurez, en relación a la ejecución de los sistemas, es imperfecto, porque hay poca confianza, un menor nivel de eficiencia, y los lapsus resaltan lo acordado. En este sentido, el objetivo de la investigación fue demostrar en qué medida un sistema experto mejora la Gestión de Servicios de TI en la Sion Global Solution Company en el tiempo esperado, los niveles de confiabilidad y la efectividad en las evaluaciones de los niveles de madurez en la gestión de servicios informáticos de la empresa Sions. La fundación del sistema se ejecutó bajo la unión de la metodología CommonKADS y SCRUM por dos aspectos destacables, que fue la reconstrucción del conocimiento y alcance del proyecto. La metodología utilizada sostiene que el enfoque cuantitativo permite la verificación de la variable que se ejecutó a través de los números que correspondían a tiempo, confidencialidad y efectividad. Asimismo, se utilizaron dos metodologías para diseñar el sistema y lograr resultados que verificaran la mejora del contexto, el diseño fue Pre-experimental en vista de que se trabajó con un solo grupo, la muestra fue determinada por 16 evaluaciones de empresas de tecnología en Perú. Los resultados mostraron que el sistema experto optimizó el tiempo promedio en la evaluación de los niveles de madurez en un 585\%, en paralelo con el sistema habitual. De esta forma, los niveles de confiabilidad optimizaron en un $98 \%$ y la eficiencia en un $113 \%$ el volumen de respuesta, la efectividad del servicio y la práctica en las evaluaciones de administración tecnológica. Se indicó que los procesos de las fases de la administración de servicios tales como delineación, táctica, transición, trabajo y mejora perenne, contribuyen en la reducción de tiempos, costos, ampliando su funcionalidad para las organizaciones que trabajan con servicios tecnológicos como parte de la maniobra del trato. Por tanto, el sistema experto podría ser utilizado en empresas de diferentes áreas que cuenten con servicios tecnológicos, cuyo objetivo es determinar el momento actual de la asistencia y su potencial mejora en el tiempo.

Palabras claves: Gestión de servicios de TI; CommonKADS; Scrum; ITIL; Sistema experto. 


\begin{abstract}
In the company, it was determined that the level of maturity, in relation to the execution of the systems, is imperfect, because there is limited confidence, a lower level of efficiency, and the lapses highlight what was agreed. In this sense, the objective of the research was to demonstrate to what extent an expert system improves IT Service Management in the Sion Global Solution Company in the expected time, the levels of reliability and the effectiveness in the assessments of the levels of maturity in the management of IT services of the company Sions. The foundation of the system was executed under the union of the CommonKADS and SCRUM methodology for two notable aspects, which was the reconstruction of the knowledge and scope of the project. The methodology used maintains that the quantitative approach allows the verification of the variable that was executed through the numbers that corresponded to time, confidentiality, and effectiveness. Likewise, two methodologies were used to design the system and achieve results that verified the improvement of the context, the design was Pre-experimental in view of the fact that it worked with a single group, the sample was determined by 16 evaluations of technology companies in Peru. The results showed that the expert system optimized the average time in assessing maturity levels by $585 \%$, in parallel with the usual system. In this way, the reliability levels optimized by $98 \%$ and the efficiency by $113 \%$ the response volume, the effectiveness of the service and the practice in the technological administration evaluations. It was indicated that the processes of the phases of the administration of services such as delineation, tactics, transition, work and perennial improvement, contribute by reducing times, costs, extending their functionality for organizations that work with technological services as part of the maneuver of the deal. Therefore, the expert system could be used in companies of different areas that have technological services, whose objective is to determine the current moment of assistance and its potential improvement over time.
\end{abstract}

Keywords: ITSM; CommonKADS; Scrum; ITIL; Expert system.

\title{
Introducción
}

Los adelantos tecnológicos se han propagado y diversificado a nivel mundial, por ser el eje de las múltiples oportunidades que contribuyen al desarrollo de las personas y organizaciones empresariales en general, a pesar de que nuestra intervención como actores indirectos no es lo suficiente. Las tecnologías son versátiles, amplias y heterogéneas, por lo que se pretende el esfuerzo, perspicacia, comprensión y apreciación de la población, porque están cambiando realidades (economía, hábitos, actividades comerciales, empresarios, expertos y tecnologías) que nos ayuda afinar el aspecto profesional y el futuro próximo de las localidades (Surdak, 2018; Sacolick, 2017).

Axelos (2019) indica que el 70\% de profesionales sustentaron que las empresas han trazado sus destrezas de forma organizada, pero sin un sentido sincerado de su avance. Es decir que, al implementar los nuevos conocimientos de tecnología, no se tiene contemplado el nivel de madurez de los procesos en las empresas y desconocen el camino a seguir para conseguir el desarrollo sostenible, en este sentido según los datos obtenidos en un estudio, el $61 \%$ de expertos registraron la importancia de organizar los objetivos transcendentales de las empresas con los valores tecnológicos del negocio (Lifeder, 2018). 
Roeder (2018); ClydeBank (2017); y Mohan (2016) reseñaron que los cálculos y establecimiento de los modelos para los acontecimientos tecnológicos fueron de gran relevancia, más aún para la gestión de servicios de TI. Es decir, ofrecen las pautas, modelos, políticas, valoran los niveles de madurez y establecen las mejores prácticas, fundamentando la ejecución de la inteligencia artificial que es ofrecida como un nuevo servicio para los procesos de las nuevas tecnologías de información.

Meléndez y Dávila (2017); Medina y Rico (2016); Mesquida, Mas y Amengual (2016) sustentaron que las empresas deben realizar el mejoramiento permanente en los procedimientos tecnológicos, el cual nos va a avalar la eficacia, siempre y cuando empleen comprobaciones para detectar los niveles de madurez en los conocimientos tecnológicos en concordancia con el modelo implementado, para la primordial relación entre servicio y negocio. Asimismo, Overton y Dixon (2019); y Cuneo (2019) registraron la importancia de valorar los niveles de madurez de los servicios de TI en las empresas públicas o privadas, con la finalidad de conocer el estado situacional y la calidad de sus tecnologías en el proceso organizacional en base a la puesta en marcha de las tecnologías básicas para la administración de servicios, determinando el grado de la importancia de la innovación digital.

A nivel nacional tenemos la contribución de varios autores que abordaron en sus averiguaciones la problemática sobre la gestión de servicios de TI, entre ellos tenemos a López y Schuler (2017); y Vásquez (2017) fortificaron la calidad de manejar varios sistemas para optimizar la calidad, eficacia, recurso, rendimiento y seguridad al momento de la ejecución, operación y valoración de los servicios tecnológicos. Por ello, nos encomienda emplear las buenas prácticas de ITIL y CMMI en los procesos de las empresas, con la intención de generar valía, orden y eficacia de servicio. Asimismo, Cruz y Lévano (2017); y Vásquez (2017) ratificaron que las organizaciones al tener gran dependencia de los conocimientos tecnológicos de información están en la obligación de provocar el progreso de sus procesos y servicios, con el fin de darle valía a la empresa a través la explotación de los datos, unificación de servicios y progreso de sus procesos de servicios.

Siongs Global Solution, compañía dedicada a la prestación servicios de tecnologías informáticas en su modalidad de outsourcing, tiene un staff de expertos para la transmisión de valor y servicio del área de tecnología, el cual pertenece al gobierno de TI. El área de gobierno muestra dificultades que están dificultando la eficiencia y eficacia a la organización. Examinando el GAP de análisis perteneciente al área de gobierno de TI, se verificaron los siguientes problemas: (a) evaluaciones incompletas; (b) bajo nivel de eficiencia y demora en la entrega de los niveles de madurez; (c) niveles de madurez inexactos; (d) incumplimiento de la alineación de los procesos; y (e) bajo nivel de confiabilidad;

Se planteó la formulación del problema de investigación: ¿En qué medida un sistema experto mejora la Gestión de servicio de la TI en la empresa Sion Global Solution?, teniendo como objetivo general: Demostrar en qué medida un sistema experto mejora la Gestión de servicio de las TI en la Empresa Sion Global Solution y como hipótesis General: Un sistema experto mejora la gestión de servicio de TI en una empresa Tecnológica. 
Páez, Rohvein, Paravie y Jaureguiberry (2018) aseveraron, que la gestión de servicios de TI debe ser ordenada y aprovechada con las mejores prácticas del mercado, manejando la metodología ITIL o ISO/IEC 20000, la que ofrecerá los juicios para todos los procesos de TI y así ofrecer la fórmula en alto nivel para poder tener claro el nivel de madurez, conveniente para el diseño, acción y evolución de los servicios de TI. Asimismo, Lia (2014), Quintero y Peña (2017) en su trabajo denominada (Modelo basado en ITIL para la Gestión de los Servicios de TI en la Cooperativa de Caficultores de Manizales) demostraron, el aumento de la calidad y el nivel de eficacia en un $60 \%$ para los servicios estratégicos, aseverando la extensión de la confidencialidad en la valor del estado actual de la empresa.

Tanovic y Mastorakis (2016) en su investigación aseveraron que, al utilizar un procedimiento inteligente basado en la gestión, se logró optimizar los tiempos de los servicios en un $35.68 \%$ a diferencia del sistema habitual, motivo por el cual lograron extender la eficacia en los servicios tecnológicos. En el contexto, los escritores como Scheruhn, Reinboth y Habel (2018) plantearon el uso de un sistema inteligente que acceda autoevaluar los procesos de la GSTI fundadas en la metodología ITIL. Teniendo en cuenta que las empresas que carecen de presupuestos para implementar y alinear los procesos de tecnologías con la estrategia del negocio. Por ello, que en sus averiguaciones explicaron la implementación de equipos que optimicen estos procesos, creando confidencialidad, eficacia, calidad, recurso, disminución de costos y rapidez para identificar las inexactitudes, y así tener un proceso maduro en el tiempo.

Yandri, Nugeraha y Zahra (2019), Jäntti y Cater (2017) aceptaron en su indagación, la utilización de una herramienta basada en ITIL, denominada ProactiveNet el cual, tiene funcionalidades para valorar la confidencialidad, recurso, eficiencia, usabilidad y calidad como parte de las actividades operacionales de ITIL. Optimizando la gestión de los servicios tecnológicos en un $45 \%$ debido a la sistematización integral de todos los procesos tecnológicos. Por otro lado, Albeti y Souza (2015) en su investigación llamada (Information Technology service management processes maturity in the Brazilian federal direct administration) ratificaron, que al monopolizar las herramientas basada en ITIL, se pudo optimizar los niveles de madurez en un $40 \%$.

En las investigaciones de Cantabella (2018); Icarte (2016); y Vilcahuamán (2016) justificaron, la importancia de emplear la inteligencia artificial y los sistemas inteligentes, porque perfeccionan los procesos tecnológicos en las organizaciones. Estos sistemas demuestran el conocimiento de un experto, la cual ha sido ingresada en la lógica de programación del sistema para predecir la conducta. Scheruhn, Reinboth, y Habel (2018) manejaron los modelos inteligentes basado en ITIL para la innovación de los procesos del centro informático de la Universidad Harz, y las conclusiones reportaron que, mediante el uso de un sistema inteligente, se logra perfeccionar grandemente en un $60 \%$ los procesos administradores de requerimientos, incidentes, cambios y problemas.

En el mismo contexto, se exponen las diferentes teorías mediante el aporte de varios académicos como De la Peña y Velázquez (2018) cuyas contribuciones dan sustento como una base extendida a las variables del estudio. Entre estas teorías tenemos: (a) la teoría del enfoque sistémico de la administración; (b) la teoría general de sistemas; y (c) la teoría situacional. Esta investigación se encamina principalmente en la teoría de sistemas. El padre de la teoría de

Esta obra se comparte bajo la licencia Creative Common Atribución-No Comercial 4.0 International (CC BY-NC 4.0) Revista de la Universidad Internacional del Ecuador. URL: https://www.uide.edu.ec/ 
sistemas Von Bertalanffy (1968) sustentó que la teoría de sistemas busca unir, juntar, integrar y relacionar los numerosos organismos, aparatos para la razón holística de funcionalidad.

En este sentido, la teoría de sistemas determina los distintos fenómenos en un enfoque globalizado, consiguiendo la unificación y relación entre sus componentes. De la Peña y Velázquez (2018) sustentaron, que la teoría general de sistemas desde una orientación organizacional comprende un conjunto de objetos que se encuentran conectados entre todos sus aparatos, y tienen un objetivo en común que se efectúa en diferentes áreas de estudio. Por tanto, la presente investigación está muy vinculada con la aplicación de los sistemas expertos que son progresos de la teoría de sistemas y que comunican todos sus tipos que se componen a diferentes recursos tecnologías.

Puri (2018) y Skillsoff (2018) concluyeron, que los sistemas expertos son sistemas o materiales informáticas que representan el comportamiento, habilidad, comprensión, y la práctica del experto humano, el cual abarca un dominio en específico. Makridakis (2018); Kornienko, Fofanov y Chubik (2015) y Klashanov (2016) refirieron, que los sistemas expertos son materiales tecnológicos que acceden a procesar, retener, aprender y motivar sobre los casos para poder solucionar los problemas que coligado a internet se tiene superioridades significativas para tomar nuevos casos empresariales. Asimismo, Mathivet (2018) y Lloret (2018) confirmaron, que los sistemas expertos son equipos tecnológicos que no solo emplean la sensatez del experto, sino también se encarga de cultivarse y acumular este nuevo conocimiento, para que sea aprovechado por los nuevos principiantes quienes podrán establecer o tomar fallos en base a los resultados obtenidos, de acuerdo con las tipos, dispositivos y tipologías que presentan.

Giarratano y Relay (2001) sostuvieron, que la estructura de un sistema experto está compuesta por lo siguiente: (a) motor de inferencia, encargado del razonamiento, además de elegir discretamente las reglas según la interacción del beneficiario final; (b) base de conocimiento, es representante de concentrar la experiencia del técnico humano a través de la lógica del sistema; (c) base de hechos, encargada de almacenar temporalmente los resultados obtenidos del proceso de evaluación. Estos mecanismos de los sistemas expertos se diferencian dependiendo del tipo de sistema de experto a utilizar.

Los SE al ser parten de la revolución digital tienden a desarrollarse con el transcurrir del tiempo y de los sucesos propuestos por el mercado tecnológico, por ello, se puede identificar varios tipos de sistemas expertos según el aporte académico de los siguientes autores como: Makridakis (2018); y Klashanov (2016) confirmaron, que los tipos de sistemas expertos están conformados de la siguiente manera: (a) basada en reglas condicionales; (b) basada en casos; (c) lógica difusa; y (d) redes neuronales. Al revisar la propuesta de cada uno de los autores, se aprobó que coexiste un nivel de complicación al instante de elegir el tipo de técnica experto, debido a que se tiene que aprobar cuál de todos se alinea mejor a la proposición de solución y la habilidad de negocio.

Meléndez y Dávila (2017) sostuvieron, que la GSTI es la apoderada de gestionar los procesos técnicos con calidad, eficiencia, confiabilidad, pacto con el requerimiento del negocio, para ello, se apoya en los siguientes componentes: (a) personas; (b) procesos; (c) tecnología; e (d) información. Atlassian (2019) y Klashanov (2016) corroboraron que existen tres mecanismos 
críticos para que la GSTI pueda desarrollarse y fructificar en el entorno de las empresas, estos componentes son: (a) las personas; (b) tecnologías; y (c) procesos, cuyo fin es la alineación valiosa con los conocimientos de la información.

La GSTI al ser implementada correctamente, se busca que brinden valor a la organización, para ello, se aplica servicios de calidad, eficacia y confidencialidad en todos los procesos de TI. Rivera (2019); Dobbins y Rov (2018) defendieron, que la gestión de servicios de tecnologías informáticas son servicios que indemnizan las necesidades del negocio, el cual es realizado por los vendedores de servicios de TI mediante el uso de conocimientos, personas y procesos, el cual tiene que ser moderado por un especialista. Asimismo, considerando la diligencia de las buenas prácticas tecnológicas para la GSTI el cual está formado por las cinco fases que contienen un conjunto de técnicas, a todo ello se designa ciclo de vida de ITIL.

\section{Metodología}

Para el presente estudio se utilizó el enfoque cuantitativo debido a las características que amerita la investigación como: (a) ser objetiva; (b) contrasta la hipótesis de investigación; (c) utiliza la estadística inferencial para el análisis de sus resultados; (d) presenta control de sus variables; y (e) por utilizar el método deductivo (Rodríguez (2017) y (Hercelinskyj y Louise (2018).

Se utilizó el tipo de investigación aplicada por que su esencia está basada en resolver problemas basada en propuesta y soluciones. Según el aporte metodológico de Hernández, Fernández y Baptista (2014) y Lifeder (2018) coinciden en que el tipo de investigación aplicada se centra en la resolución de problemas, más aún por tener un lineamiento de diseño experimental. En el mismo contexto Sánchez, Reyes y Mejía (2018) sostienen que la evaluación y resolución de problemas basadas en soluciones, es parte de los tipos de investigación aplicada, además por su amplia vinculación por la investigación del tipo básica por generar conocimiento en base a los aportes.

Respecto al diseño de investigación, se planteó un diseño de tipo experimental Pre experimental porque se realizó la manipulación de la variable independiente para poder revisar y validar su reacción frente al efecto aplicado. Es decir, se manipulo la variable sistema experto mediante el uso de un software personalizado denominado IT Assesment en la variable gestión de servicios de tecnologías de información, donde se verificó su efecto. Según Flores, Carhuancho, Venturo, Sicheri y Mendivel (2019); Carhuancho, Nolazco, Sicheri, Guerrero y Casana (2019); Solíz (2019) sostienen utilizando un diseño pre experimental se logra conocer el efecto de una variable con otra, más aún si los factores que logran su cometido son las dimensiones tiempo, eficiencia y confiabilidad.

La investigación utilizada fue un enfoque cuantitativo con un diseño pre experimental, y se optó por utilizar el método deductivo, el cual permite su aplicación desde una perspectiva general, para luego profundizar el estudio en algo más específico. Es decir, se aplicó para un grupo de la población una prueba pretest utilizando el sistema tradicional y luego una prueba postest utilizando el sistema experto implementado. 
La población de estudio estuvo conformada por 16 evaluaciones de madurez de la gestión de TI, el cual se llevó a cabo en una empresa tecnológica para el primer semestre del año 2019. Según Hernández, Fernández y Baptista (2014); Flores y Mendivel (2019) sostienen que la población es un conjunto de elementos que poseen características temporales, espacio y tiempo. Asimismo, reitera que los autores pueden optar, de no utilizar una muestra de estudio, siempre y cuando plasmen en su investigación el uso de todos los elementos de su población. Ante esta premisa, la población de estudio estuvo conformada por 16 evaluaciones para el pretest y postest en la gestión de TI en una empresa tecnológica.

La técnica y los instrumentos de recolección de datos que permitieron la realización de la presente investigación se basan en los aportes prácticos de Flores et al (2019) y Guillen y Valderrama (2015), ambos recomiendan el uso de la técnica de la observación para poder registrar la información sobre los temas de estudio, sin la intervención del participante. Sobre los instrumentos de recolección, se utilizaron fichas de observación durante todos los 10 procesos de la gestión de TI basadas en la operación y diseño del servicio, con el fin de poder recopilar, revisar y analizar los niveles de madurez.

Los datos recopilados mediante las fichas de observación fueron instrumentos que previamente fueron validados por expertos en Ingeniería de sistemas y expertos en la gestión de tecnologías de información en empresas de tecnología del Perú. Asimismo, para revalidar su validez y confiabilidad del instrumento se aplicaron pruebas piloto, para validar su consistencia. Para culminar, se utilizó la estadística descriptiva e inferencial la contrastación de las hipótesis de investigación basas en las dimensiones tiempo, eficiencia y confiabilidad.

Rezault (2019), Llinás (2018), Dzul (2018) y Supo (2014) coinciden que la estadística inferencial debe encaminarse con una secuencia de pasos como: (a) consistencia de datos; (b) prueba de normalidad; (c) significancia; y (d) constratacion de hipótesis.

\section{Resultados}

En el presente estudio, se consideró el uso de los estadísticos descriptivos e inferencial como parte de la estructura de los resultados. Para ello, se utilizó los 3 indicadores mediante la aplicación del sistema experto con el fin de evaluar la gestión de tecnologías de información antes y después. Sin utilizar el sistema experto de evaluación los resultados fueron los siguientes: (a) el indicador tiempo presento un valor máximo de 736 minutos y un mínimo de 707 minutos, con una media de 726 minutos aproximados, con una desviación estándar de 7,08 y una variación de 50.16.; (b) el indicador confiabilidad evidencio un máximo del 54.55\% y un mínimo del $45.45 \%$, la media fue del $50.56 \%$ aproximadamente, con una desviación del $4.56 \%$ y una varianza del 21,7\%; y (c) El indicador eficiencia evidencio un máximo del $47.43 \%$ y un mínimo del $34.49 \%$, la media fue de $43.49 \%$ aproximadamente, con una desviación del $4 \%$ y una varianza del 16,35. Es decir los tiempos de la evaluación sin utilizar el sistema tradicional aun presenta tiempos elevados, generando que se requiere de más especialistas en proceso de ITIL, para las evaluaciones en las empresas tecnológicas. Aunado a ello, se puede evidenciar la eficiencia y la confiabilidad el cual aún es muy baja, generando re trabajos, evaluaciones 
incompletas y costos excesivos por la extensión de los tiempos en las evaluaciones de madures de los servicios. Debido a esta problemática, se implementó el uso de un sistema experto para las evaluaciones.

En el postest, utilizando el sistema experto como parte de la estadística descriptiva, se obtuvo los siguientes resultados: (a) el indicador tiempo presento un máximo de 107 minutos y un mínimo de 105 minutos. Es decir, los tiempos disminuyeron en 629 minutos aproximadamente; (b) el indicador confiabilidad evidencio un máximo de $100 \%$ y un mínimo de 99.99\%. Es decir, se mejoró la confiabilidad en un $45 \%$ aproximadamente respecto al pretest; y (c) la eficiencia presento un valor máximo de $94.34 \%$ y un mínimo de $93.40 \%$. Es decir, se mejoró la eficiencia en una $46 \%$ aproximadamente, todo ello se puede apreciar en la tabla 1.

\section{Tabla 1}

Consolidado de los indicadores tiempo, confiabilidad y eficiencia

\begin{tabular}{ccccccc}
\hline Empresa & $\begin{array}{c}\text { Tiempo } \\
\text { (Pre-Test) }\end{array}$ & $\begin{array}{c}\text { Tiempo } \\
\text { (Post-Test) }\end{array}$ & $\begin{array}{c}\text { Confiabilidad } \\
\text { (Pre-Test) }\end{array}$ & $\begin{array}{c}\text { Confiabilidad } \\
\text { (Post-Test) }\end{array}$ & $\begin{array}{c}\text { Eficiencia } \\
\text { (Pre-Test) }\end{array}$ & $\begin{array}{c}\text { Eficiencia } \\
\text { (Post-Test) }\end{array}$ \\
\hline Empresa 1 & 731 & 105 & $54.55 \%$ & $100 \%$ & $47.38 \%$ & $94.29 \%$ \\
Empresa 2 & 725 & 106 & $45.45 \%$ & $100 \%$ & $39.50 \%$ & $94.34 \%$ \\
Empresa 3 & 725 & 106 & $54.55 \%$ & $100 \%$ & $47.40 \%$ & $93.40 \%$ \\
Empresa 4 & 736 & 106 & $54.55 \%$ & $100 \%$ & $47.43 \%$ & $93.40 \%$ \\
Empresa 5 & 731 & 106 & $45.45 \%$ & $100 \%$ & $39.49 \%$ & $94.34 \%$ \\
Empresa 6 & 731 & 106 & $54.55 \%$ & $100 \%$ & $47.38 \%$ & $93.40 \%$ \\
Empresa 7 & 707 & 107 & $45.45 \%$ & $100 \%$ & $39.54 \%$ & $93.46 \%$ \\
Empresa 8 & 725 & 105 & $45.45 \%$ & $100 \%$ & $39.50 \%$ & $94.29 \%$ \\
Empresa 9 & 731 & 106 & $54.55 \%$ & $100 \%$ & $47.38 \%$ & $93.40 \%$ \\
Empresa 10 & 731 & 107 & $45.45 \%$ & $100 \%$ & $39.49 \%$ & $93.46 \%$ \\
Empresa 11 & 725 & 106 & $54.55 \%$ & $100 \%$ & $47.40 \%$ & $94.34 \%$ \\
Empresa 12 & 731 & 106 & $54.55 \%$ & $100 \%$ & $47.38 \%$ & $93.40 \%$ \\
Empresa 13 & 731 & 105 & $45.45 \%$ & $100 \%$ & $39.49 \%$ & $94.29 \%$ \\
Empresa 14 & 731 & 106 & $54.55 \%$ & $100 \%$ & $47.38 \%$ & $93.40 \%$ \\
Empresa 15 & 719 & 105 & $45.45 \%$ & $100 \%$ & $39.51 \%$ & $94.29 \%$ \\
Empresa 16 & 719 & 106 & $54.55 \%$ & $100 \%$ & $47.41 \%$ & $93.40 \%$ \\
\hline
\end{tabular}

Como parte de la estadística inferencial, se realizó la consistencia de los datos recopilados mediante la prueba de doble de masas, el cual se aplicó para los 3 indicadores (a) indicador tiempo; (b) indicador confiabilidad y (c) indicador eficiencia, tanto para el pretest y post test. El resultado obtenido fue favorable donde se pudo apreciar que si existe consistencia en los datos acumulados. Para la prueba de normalidad se utilizó Shapiro-Wilk donde se evidencio que los datos son no paramétricos. Los resultados fueron consolidados y evidenciados en la tabla 2 


\section{Tabla 2}

Consolidado de los indicadores tiempo, confiabilidad y eficiencia - Shapiro Wilk

\begin{tabular}{lccc}
\hline & $\begin{array}{c}\text { Shapiro-Wilk } \\
\text { Estadístico }\end{array}$ & gl & Sig. \\
\hline Tiempo (Pre) &, 806 & 16 &, 003 \\
Tiempo (Post) &, 778 & 16 &, 001 \\
Confiabilidad (Pre) &, 638 & 16 &, 000 \\
Confiabilidad (Post) &, 273 & 16 &, 000 \\
Eficiencia (Pre) &, 642 & 16 &, 000 \\
Eficiencia (Post) &, 676 & 16 &, 000 \\
\hline
\end{tabular}

En la aplicación de la prueba de contraste se utilizó la prueba de rangos de Wilcoxon para los 3 indicadores, donde se lograron los siguientes resultados:

$\mathrm{El}$ indicador tiempo presento un $\mathrm{Zc}=3.531 \mathrm{y}$ un $\mathrm{p}$ valor $=.000$, por esta razón la hipótesis nula se rechaza y se confirma la aceptación de la hipótesis de investigación donde el sistema experto reduce el tiempo de evaluación en la gestión de servicios de TI. Todo ello se comprueba en la figura 1, donde el tiempo disminuyo considerablemente en 621 minutos, es decir representa una mejora de $586 \%$.

\section{Figura 1}

\section{Indicador tiempo}

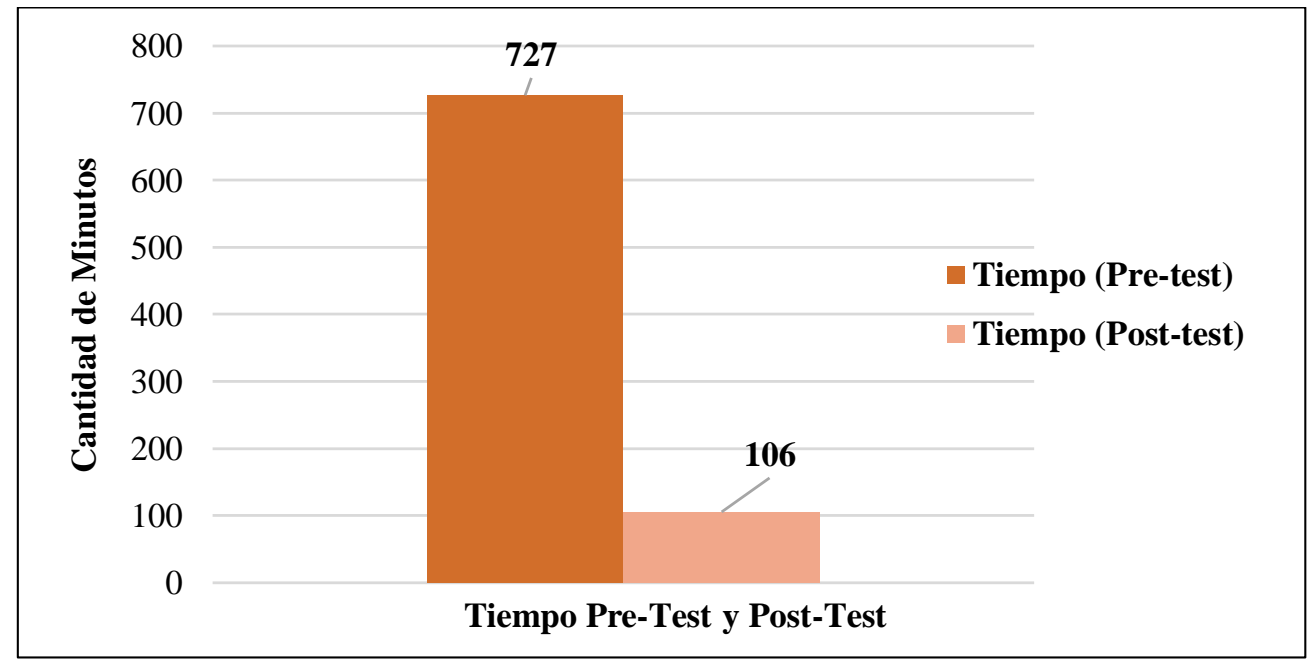

El indicador confiabilidad presento un Zc $=3.601$ y un p valor $=.000$, por esta razón la hipótesis nula se rechaza y se confirma la aceptación de la hipótesis de investigación donde el sistema experto incrementa la confiabilidad en la evaluación de la gestión de servicios de TI. 
Todo ello se comprueba en la figura 2, donde la confiabilidad se incrementó considerablemente en un $49 \%$ aproximadamente, es decir representa una mejora del $98 \%$.

\section{Figura 2}

\section{Indicador confiabilidad}

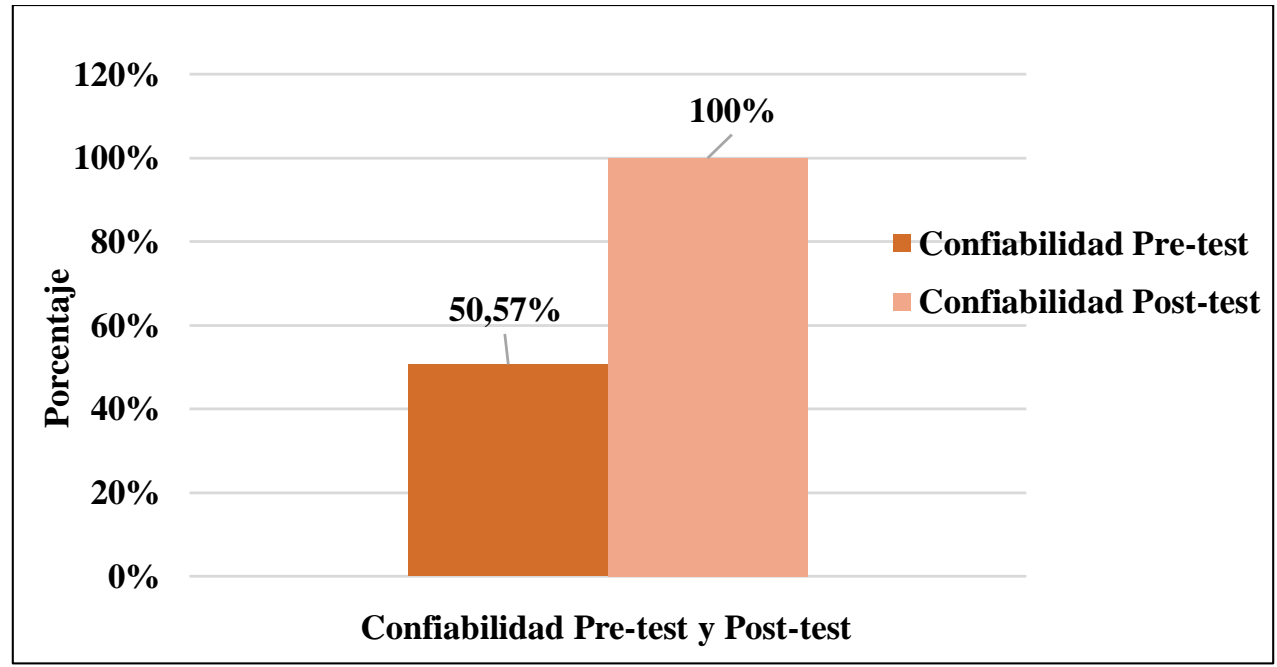

El indicador eficiencia presento un $\mathrm{Zc}=3.522 \mathrm{y}$ un $\mathrm{p}$ valor $=.000$, por esta razón la hipótesis nula se rechaza y se confirma la aceptación de la hipótesis de investigación donde el sistema experto incrementa la eficiencia en la evaluación de la gestión de servicios de TI. Todo ello se comprueba en la figura 3, donde la eficiencia se incrementó considerablemente en un 49\% aproximadamente, es decir representa una mejora del $113 \%$.

\section{Figura 3}

\section{Indicador eficiencia}

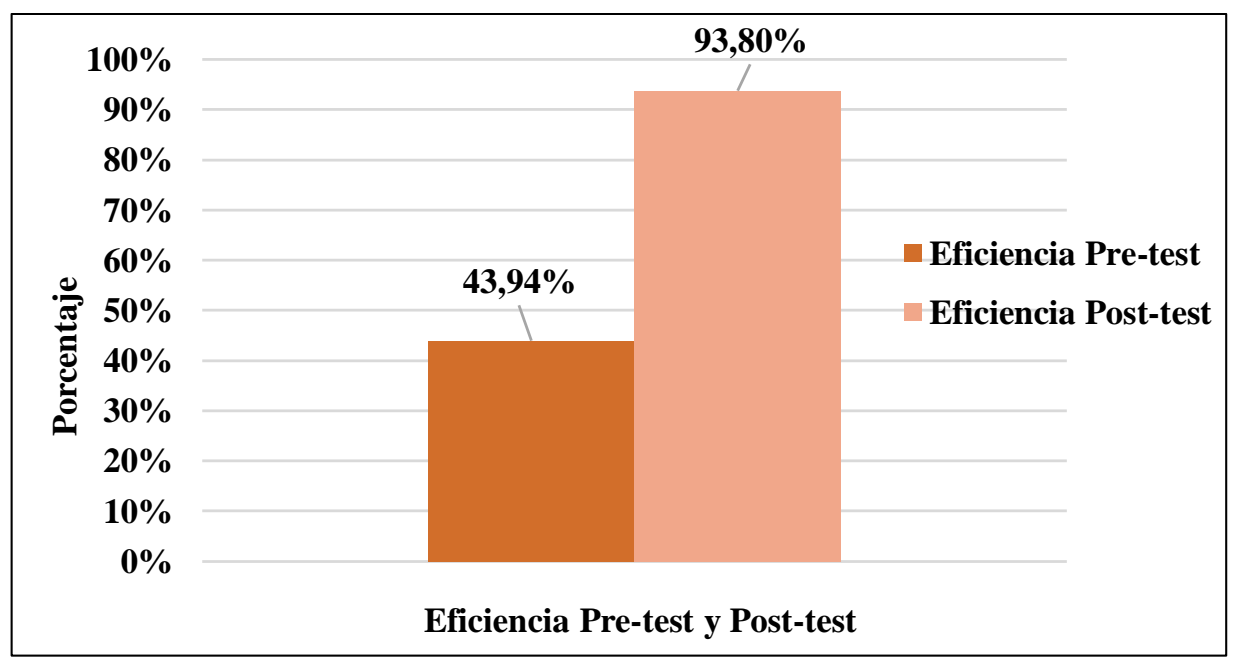




\section{Conclusiones}

Con la implementación del sistema experto, el primer objetivo respecto al tiempo se redujo el promedio de las evaluaciones en 106 minutos; y sin utilizar el sistema experto, el tiempo de evaluación fue de 727 minutos, obteniéndose una disminución considerable de 621 minutos. Es decir, una disminución del 586\% del tiempo. Todo ello. Contribuye con la mejora, debido a que la empresa tecnológica, antes realizaba 3 IT Assesment en 36 horas aproximadamente, y ahora realiza 20 IT Assesment para el indicador tiempo.

El segundo objetivo, con la implementación del sistema experto se mejoró la confiabilidad promedio de las evaluaciones en 100\%; y sin utilizar el sistema experto, la confiabilidad de la evaluación fue del $50.57 \%$, obteniéndose un incremento considerable del $49 \%$ aproximadamente. Es decir, un incremento del $98 \%$ en la confiabilidad. Todo ello contribuye con la mejora, debido a que la empresa tecnológica, antes realizaba el 50\% IT Assesment confiables, y ahora realiza el 100\% de IT Assesment confiables aproximadamente.

El tercer objetivo, con la implementación del sistema experto se mejoró la eficiencia promedio de las evaluaciones en $93.80 \%$; y sin utilizar el sistema experto, la eficiencia de la evaluación fue de $43.94 \%$, obteniéndose un incremento considerable del $49 \%$ aproximadamente. Es decir, un incremento del $113 \%$ en la eficiencia. Todo ello contribuye con la mejora, debido a que la empresa tecnológica, antes realizaba el 50\% IT Assesment confiables, y ahora realiza el 94\% de IT Assesment confiables aproximadamente.

\section{Referencias bibliográficas}

Albeti, V., \& Souza, J. (2015). Information Technology service management processes maturity in the Brazilian federal direct administration. Journal of Information Systems and Technology Management, 663-686.

Atlassian. (2019, mayo 05). Atlassian.com. Retrieved from es.atlassian.com: https://es.atlassian.com/it-unplugged/itsm

Axelos. (2019, mayo 04). Axelos.com. Retrieved from https://www.axelos.com: https://www.axelos.com/best-practice-solutions/itil

Cantabella, M. (2018). Modelos y herramientas para la representación y análisis de datos en LMS para enseñanzas universitarias. Murcia: Escuela internacional de Doctorado UCM.

Carhuancho, I., Nolazco, F., Sicheri, L., Guerrero, M., \& Casana, K. (2019). Metodología de la investigación holística. Guayaquil, Ecuador: UIDE.

ClydeBank. (2017). ITSM QuickStart Guide: The Simplified Beginner's Guide to IT Service Management. New York: ClydeBank Media LLC.

Cruz, J., \& Lévano, D. (2017). Nivel de Madurez de los procesos de la gestión de servicios en base a BPM. Redalyc.org, 13. Retrieved from http://www.redalyc.org: http://www.redalyc.org/pdf/4676/467646123008.pdf 
Cuneo, K. (2019, junio 2). yourlearning.ibm.com. Retrieved from yourlearning.ibm.com: https://yourlearning.ibm.com/\#search/activities/q=maturity

De la Peña, G., \& Velázquez, R. (2018). Algunas reflexiones sobre la teoría general de sistemas y el enfoque sistémico en las investigaciones científicas. Revista Cubana de Educación Superior, 14.

Dobbins, C., \& Rov, R. (2018). Gestão de Projetos em Tecnologia da Informação: Estudo de Caso sobre a Implementação e Avaliação Desta Ferramenta em Fundo de Investimento Multimercado. Revista de Tecnologia Aplicada, 36-51. doi: http://dx.doi.org/10.21714/2237-3713rta2018v7n3p36

Dzul, M. (2019, enero 19). www.uaeh.edu.mx. Retrieved from https://www.uaeh.edu.mx: https://www.uaeh.edu.mx/docencia/VI_Presentaciones/licenciatura_en_mercadotecnia/fu ndamentos_de_metodologia_investigacion/PRES39.pdf

Flores, D., \& Mendivel, I. (2019). Sistema experto para mejorar la salud nutricional mediante la evaluación y recomendación de dietas nutricionales. Tlatemoani, 32, 19-30. Retrieved from https://www.eumed.net/rev/tlatemoani/32/dietas-nutricionales.html

Flores, D., Carhuancho, I., Venturo, C., Sicheri, L., \& Mendivel, I. (2019). Expert System for Information Technology Services Management. International Journal of Recent Technology and Engineering, 9986-9992.

Giarratano, J., \& Relay, G. (2001). Sistemas Expertos: Principio y programación. Madrid, España: Editorial Internacional Thompson.

Guillen, O., \& Valderrama, S. (2015). Guía para elaborar la tesis universitaria escuela de posgrado. Lima: Ando Educando.

Hercelinskyj, G., \& Louise, A. (2018). Mental Health Nursing: Applying Theory to Practice. South Melbourne: Cenveo Publisher Services.

Hernández, R., Fernández, C., \& Baptista, P. (2014). Metodología de la Investigación. Distrito Federal: McGraw-Hill.

Icarte, G. (2016). Aplicaciones de inteligencia artificial en procesos de cadenas de suministros: una revisión sistemática. Iquique: Revista chilena de ingeniería. doi: http://dx.doi.org/10.4067/S0718-33052016000400011

Jäntti, M., \& Cater, A. (2017). Proactive management of IT Operations to improve it services. Journal of Information Systems and Technology Management, 191-218. doi: 10.4301/S1807-17752017000200004

Klashanov, F. (2016). Artificial Intelligence and Organizing Decision in Construction. Procedia Engineering., 1016-1020. doi: https://doi.org/10.1016/ j. proeng.2016.11.813

Kornienko, A., Fofanov, O., \& Chubik, M. (2015). Knowledge in artificial intelligence systems: searching the strategies for application. Procedia - Social and Behavioral Sciences, 589594.

Lia, R. (2014). ITSM: um caso de sucesso do modelo Tríplice Hélice. Revista de Administração da UFSM, 15. doi:10.5902/1983465911460

Esta obra se comparte bajo la licencia Creative Common Atribución-No Comercial 4.0 International (CC BY-NC 4.0) Revista de la Universidad Internacional del Ecuador. URL: https://www.uide.edu.ec/ 
Lifeder. (2018, diciembre 21). https://www.lifeder.com. Retrieved from lifeder.com: https://www.lifeder.com/investigacion-aplicada/

Llinás, H. (2018). Estadística Inferencial. Barranquilla: Universidad del Norte.

Lloret, J. (2018, enero 25). Linkedin.com. Retrieved from https://es.linkedin.com: https://es.linkedin.com/pulse/sistemas-expertos-en-el-diagn\%C3\%B3sticom\%C3\%A9dico-expert-systems-lloret

López, O., \& Schuler, J. (2017). Implementación de buenas prácticas de CMMI - SVC e ITIL para la gestión de servicios de TI en la Pyme Agile Solutions. Lima: Facultad de Ingeniería y Arquitectura.

Makridakis, S. (2018). The forthcoming Artificial Intelligence (AI) revolution: Its impact on society and firms. Elsevier Ltd., 46-60. doi: https://doi. org/10.1016/j.futures. 2017.03.006

Mathivet, V. (2018). Inteligencia Artificial para desarrolladores. Barcelona: Ediciones ENI.

Medina, Y., \& Rico, D. (2016). Modelo de gestión basado en el ciclo de vida del servicio de la Biblioteca de Infraestructura de Tecnologías de Información (ITIL). Revista Virtual Universidad Católica del Norte, 23.

Meléndez, K., \& Dávila, A. (2017). Problemas en la adopción de modelos de gestión de servicios de tecnologías de información. Una revisión sistemática de la literatura. Revista DYNA, 8.

Mesquida, A., Mas, A., \& Amengual, E. (2016). La madurez de los servicios TI. REICIS. Revista Española de Innovación, Calidad e Ingeniería del Software, 11.

Mohan, S. (2016). BP Introduction to IT Service Management. UK: Learning Technologies.

Overton, L., \& Dixon, G. (2019). Unlocking Potential: Releasing the Potential of the Business and its People Through Learning. 2016-17 Towards Maturity Industry Benchmark Report. New York: Towards Maturity.

Páez, G., Rohvein, C., Paravie, D., \& Jaureguiberry, M. (2018). Revisión de modelos de madurez en la gestión de los procesos de negocios. Revista chilena de ingeniería, 14.

Puri, R. (2018). Artificial Intelligence. New York: Blueprint. doi: https://doi.org/10.1201/9781315137773

Quintero, L., \& Peña, H. (2017). Modelo basado en ITIL para la Gestión de los Servicios de TI en la Cooperativa de Caficultores de Manizales. Manizales: Universidad Autónoma de Manizales.

Rezaul, I. (2019). Social Research Methodology and New Techniques in Analysis, Interpretation, and Writing. Hershey: IGI Global.

Rivera, C. (2019). Aplicación ITIL y su efecto en la gestión de resolución de incidencias en el área de soporte de la empresa MDP consulting. Lima: Repositorio UCV.

Rodríguez, M. (2017, Julio 20). Diferencia entre investigación cualitativa y cuantitativa. Lima, Lima, Perú.

Roeder, A. (2018). eBook: Simplify IT Support Management with IBM Technology Support Services. New York: IBM Technology Support Services. 
Sacolick, I. (2017). Driving Digital: The Leader's Guide to Business Transformation Through Technology. New York: Força Digital.

Sánchez, H., Reyes, C., \& Mejía, K. (2018). Manual de términos en investigación científica, tecnológica y humanística. Lima: Vicerrectorado de Investigación Universidad Ricardo Palma.

Scheruhn, H., Reinboth, C., \& Habel, T. (2018). Einsatz von ITIL zur Prozessoptimierung im Rechenzentrum der Hochschule Harz am Beispiel des Release Managements. Friedrichstr: Hochschule Harz.

Skillsoff. (2018). Introduction to Artificial Intelligence. New York: E-learning Support.

Solíz, D. (2019). Cómo hacer un Perfil Proyecto de Investigación Científica. Bloomington: Palibrio.

Surdak, C. (2018). A Revolução Digital. São Paulo, Brasil: DVS Editora. Retrieved from https://www.getabstract.com/en/summary/a-revolucao-digital/35162?u=ibm_corporation

Tanovic, A., \& Mastorakis, N. (2016). Advantage of using Service Desk Management Systems in real organizations. International Journal of Economics and Management Systems, 1(ISSN: 2367-8925), 81-86.

Vásquez, E. (2017). Sistema experto para la gestión de incidentes de TI. Lima.

Vilcahuamán, R. (2016). Sistema inteligente para la supervisión y Monitoreo de la calidad del servicio eléctrico. Callao: Sección de posgrado de la facultad de ingeniería Universidad Nacional del Callao.

Von Bertalanffy, L. (1968). Teoría General de los Sistemas. México: Fondo de cultura económica.

Yandri, R., Nugeraha, D., \& Zahra, A. (2019). Evaluation Model for the Implementation of Information Technology Service Management using Fuzzy ITIL. Procedia Computer Science, Volume 157(10.1016/j.procs.2019.08.169), 290-297. 